\title{
HORS CADRE
}




\section{LA SOCIALISATION, ENTRE FAMILLE ET ÉCOLE}

OBSERVATION D'UNE CLASSE DE PREMIÈRE ANNÉE DE MATERNELLE

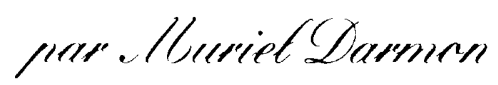

Peut-on étudier la socialisation scolaire dès l'école maternelle' ? Dans cet univers " pré-scolaire ", la place de l'écrit et de son apprentissage sont très réduites, et la ritualisation scolaire ne se présente pas sous les formes qu'elle prendra pourtant peu après ; cette particularité est encore plus nette en ce qui concerne la première année du cursus en école maternelle, la classe des «petits ». Pourtant, cette entrée détournée est en même temps une entrée privilégiée dans l'étude de la socialisation scolaire : c'est à ce stade du cursus que les enfants sont préparés à devenir des élèves, et donc que le processus de socialisation propre à l'école est le plus visible. De plus, les réquisits non scolaires de l'école s'y donnent à voir avec plus d'évidence, car l'apprentissage y apparaît comme plus social que scolaire et la confrontation entre les deux instances de socialisation que sont l'école et la famille s'y fait sous le rapport le plus " large " possible ${ }^{2}$. Moins que la socialisation familiale (qui n'a pas été étudiée ici en tant que telle) ou que la socialisation scolaire, c'est le rapport qu'elles entretiennent qui constitue l'objet de ce travail : ce qui a trait à la rencontre, au sein de l'école maternelle, des deux instances de socialisa-

1. L'enquête présentée ici a été réalisée dans le cadre du séminaire "Les usages sociaux du corps ", organisé au Département de Sciences Sociales de l’École Normale Supérieure par Frédérique Matonti et Dominique Memmi. Je remercie Frédérique Matonti, qui a dirigé le mémoire secondaire pour le DEA de Sciences Sociales (ENS-EHESS) dont est tiré cet article, ainsi qu'Anne Paillet et Delphine Serre pour leur lecture attentive.

2. $C f$. Jean-Claude Chamboredon, Jean Prévot, «Le métier d'enfant. Définition sociale de la prime enfance et fonctions différentielles de l'école maternelle », Rev'ue Française de Sociologie. $\mathrm{n}^{\circ} \mathrm{XIV}, 1973$, pp. 295-335, [p. 324]. 
tion que sont l'école et la famille, et l'étude de la façon dont elles se complètent, se superposent ou s'opposent.

L'observation semblait la méthode requise pour mettre au jour les processus des apprentissages. J'ai effectué deux périodes d'observations en continu (l'une en décembre et l'autre en mars) dans la "petite section »(première année) d'une école maternelle parisienne, au public socialement diversifié mais en majorité populaire. Très vite, ma présence dans la classe a été perçue et décrite comme un « stage », d'abord " un stage d'observation " puis un « stage » tout court. Devenant ainsi la "petite stagiaire de Catherine [l'institutrice] ",j'ai réglé mes activités et mes horaires sur les siens, « observant les activités pédagogiques » en classe et y « participant » (à sa demande), l'accompagnant en salle des maîtres ou dans la cour de récréation.

\section{L'EMPRISE DE L'APPARTENANCE SOCIALE}

La façon la plus évidente d'aborder la question des relations entre socialisations scolaire et familiale consiste à observer comment une variable unique, l'origine sociale, affecte le rapport des enfants et des parents à l'école, et à examiner les rendements différentiels de cette origine dans l'espace scolaire, à travers notamment les jugements professoraux sur les élèves et leurs travaux. L'intérêt de cette approche est évident, socialement autant que sociologiquement. D'une part, l'école maternelle a souvent été décrite comme une structure pré-scolaire pouvant compenser les « handicaps socio-culturels » des classes populaires'. D'autre part, il peut être sociologiquement judicieux d'étudier les inégalités face à l'école dans un univers sans notes ni orientation comme la première année de l'école maternellet.

3. Ce discours est rapporté par Geneviève Dannepond, "Pratiques pédagogiques et classes sociales : étude comparée de trois écoles maternelles", Actes de la recherche en sciences sociales, n 30, nov. 1979, pp. 31-45, [p. 31], et par Jean-Claude Chamboredon, “Étude critique ", Revue Française de Pédagogie, ${ }^{\circ}$ 83, avril-mai-juin 1988, pp. 83-124, [p. 83].

4. La spécificité la plus patente de l'école maternelle tient sans doute à son caractère non obligatoire, puisque l'obligation scolaire ne commence qu'à six ans. Actuellement, la quasi-totalité des enfants ayant trois ans révolus fréquente une école maternelle ou une classe enfantine. Mais des variations significatives interviennent dans le cas des enfants de deux à trois ans. L'école maternelle accueille un tiers des enfants de deux ans. Mais si, à trois ans, les enfants de cadres sont les plus scolarisés, « à deux ans ils le sont moins que les enfants d'employés et, dans l'agglomération parisienne, que les enfants d'ouvriers », Guy Desplanques, « Modes de garde et scolarisation des jeunes enfants ", Économie et Statistique, $\mathrm{n}^{\circ} 176,1985$, pp. 27-40, [p. 32]. Guy Desplanques donne deux raisons princıpales à cet état de fait, l'une historique ( « Le développement des écoles matemelles à la fin du siècle dernier répondait à un souci de protection sociale plus que d'éducation; elles devaient accueillır en priorité les enfants des couches populaires », p. 32), l'autre économique ( « Pour un enfant de deux ans. l'école maternelle est une solution moins coûteuse que la crèche ou que la nourrice ", p. 32). Dans la classe observée, la valorisation des enfants qui « ont le bon âge ", c'est-à dire qui ne sont pas rentrés "trop jeunes", participe donc de la valorisation d'un certain type de stratégies familiales. 
Même dans cet univers "pré-scolaire ", les classements scolaires n'opèrent pas moins, et ils semblent refléter dans une grande mesure des classements sociaux. On peut l'observer ici à trois niveaux'.

\section{LA PARTICIPATION}

Dans les processus de construction du bon et du mauvais élève de maternelle, le jugement sur « la participation » joue comme critère central d'évaluation. Il y a " ceux qui participent » et " ceux qui ne participent pas », au regroupement surtout", mais également lors des divers ateliers. Ce que l'institutrice désigne par le terme de "participation », c'est une prise de parole ou une gestuelle valorisées parce qu'elles manifestent l'intégration des règles du jeu scolaire, par opposition d'une part à une activité jugée désordonnée, et d'autre part à ce qui est perçu comme signe d'incapacité, de passivité ou de désintérêt. Par exemple, l'institutrice demande aux enfants « ce que c'est que la nuit » pour eux et s'inspire des réponses de « ceux qui participent » pour définir les consignes d'un dessin sur la nuit que tous devront réaliser. Le nom même de "pédagogie inductive », employé et revendiqué par l'institutrice comme l'emblème de son engagement dans une pédagogie moderne, est très explicite : puisqu'il s'agit de " travailler à partir du savoir des enfants », il est clair que cette induction s'appuie sur les acquis de la socialisation familiale'.

\section{MÉTIER D'ENFANT, MÉTIER DE PARENT}

Dans les travaux d'enfants (dessins, peintures, collages, objets divers en terre glaise ou en pâte-à-sel), l'origine sociale intervient à plus d'un titre. Tout d'abord, les enfants d’origine moyenne ou supérieure peuvent réutiliser des compétences acquises au sein de la famille pour comprendre les instructions de l'institutrice et

5. Pour un débat autour de la question des « affinités électives » entre le modèle éducatif de l'école maternelle et un certain type de socialisation familiale, $c f$. Jean-Claude Chamboredon, Jean Prévot, "Le métier d'enfant... " loc. cit. ; Éric Plaisance, L'Enfant, la maternelle, la société, Paris, PUF, 1986. deuxième partie, pp. 113-204, et la note critique que Jean-Claude Chamboredon consacre à l'ouvrage d’Éric Plaisance dans la Revue Française de Pédagogie, «Étude critique ». loc. cit.

6. Le « regroupement», qui commence avec l'arrivée de l'institutrice le matin, constitue le moment le plus scolairement ritualisé de la journée : l'institutrice y rassemble les enfants, fait l'appel, lance un thème de discussion, etc... C'est un moment « sérieux » qui préfigure la forme scolaire ultérieure.

7. Une analyse systématique de cette question de la participation pour l'école primaire se trouve dans l'étude de Régine Sirota, L'École primaire au quotidien, Paris, PUF, 1988, à partir de l'observation des interactions entre instituteurs et enfants. La valorisation de la participation enfantine s'inscrit dans ce que Éric Plaisance nomme le « modèle expressif de l'école maternelle moderne ". qui est " en concordance avec le modèle culturel des couches intellectuelles des classes moyennes et supéricures ", Éric Plaisance, L'Enfant, la maternelle, la société, op. cit., p. 200. 
réaliser les objets demandés ${ }^{8}$. De plus, les catégories de perception que les parents vont appliquer à ces réalisations, ou que l'institutrice leur attribue, influent sur les jugements portés et donc sur la construction de l'excellence scolaire.

L'article fondateur de Jean-Claude Chamboredon et Jean Prévot insistait déjà sur ce point. Il montrait en effet comment l'art contemporain et sa connaissance pouvait concourir à l'invention d'un « art enfantin », en « donnant une grille de lecture qui fait voir (...) un Picasso, un Rousseau ou une sorte de Mirò (...) là où l'on ne voyait auparavant qu'un barbouillage $"$. De fait, l'un des panneaux de la classe s'organise autour de dessins d'enfants légendés : « Nous jouons avec les formes, les couleurs et les mouvements comme... Sonia Delaunay. »

Mais « l'artisanat » (authenticité, fraîcheur, naïveté...) peut jouer le même rôle que l'art abstrait dans la valorisation de l'esthétique enfantine. Ainsi, lors de la réalisation des coquetiers en terre glaise, pour Pâques, les consignes sont très claires sur " comment on fait un coquetier » : un rouleau de terre glaise, qu'on étrangle au milieu, avant de l'évider en haut « pour faire la maison de l'oeuf ». Il s'agit d'un exercice difficile pour les enfants, beaucoup n'y parviennent pas et l'institutrice leur fait recommencer en leur montrant à chaque fois que l'œuf ne rentre pas, ou que le coquetier ne tient pas debout. Mais lorsque Lara arrive pour montrer à l'institutrice ce qu'elle a fait (un tube de terre glaise, qui ne tient pas vraiment debout, et où aucun œuf ne rentrerait), elle s'exclame : « Lara, tu as fait un magnifique bougeoir breton ! ». Une telle appréciation s'inscrit dans la présupposition que ce qu'elle voit comme " magnifique bougeoir breton » va être également vu comme tel par les parents de Lara (son père est responsable informatique, et sa mère une « amie de la directrice »), et non comme un coquetier raté.

\section{LA « PRÉSENCE » : UNE CATÉGORIE DE JUGEMENT}

La catégorie de " présence " constitue un critère de jugement qui s'applique indissociablement aux parents et aux enfants. Elle désigne tout d'abord un certain rapport des enfants à l'école, une visibilité valorisée qui rejoint la catégorie de participation («il est très présent au regroupement » signifie que l'enfant « participe bien »). Les parents apparaissent ici en creux comme les responsables, au sens fort, de l'éducation des enfants (les enfants sont ce que les parents en ont fait) ${ }^{\circ}$. Une

8. Cf. Geneviève Dannepond, " Pratiques pédagogiques et classes sociales... ", loc. cit., pp. 3941, sur la compréhension des consignes de dessin et l'intériorisation de la " norme d'ennchissement " des dessins, et Jean-Claude Chamboredon, Jean Prévot, "Le métier d'enfant... ", loc. cit., pour un traitement général de la question.

9. Jean-Claude Chamboredon, Jean Prévot, « Le métier d'enfant... », loc. cit., pp. 315-316.

10. Jean Claude Chamboredon et Jean Prévot évoquent « le moralisme qui pénètre la relation parents/institutrice. Le jugement, explicite ou en acte, sur l'enfant est très directement jugement sur les méthodes d'éducation et les éducateurs qui l'ont "produit" ", "Le metier d'enfant... ". loc. cit., p. 324, note 56 . 
deuxième occurrence possible du terme de "présence »- la présence physique des enfants - les implique encore plus directement. Il y a ainsi une bonne présence des enfants (le matin, c'est-à dire lors des activités les plus marquées par le travail pédagogique) et une mauvaise, intermittente, qui marque une non-reconnaissance par les parents de l'importance de la continuité du travail pédagogique sur l'enfant, ou, l'après-midi uniquement, qui signifie pour l'institutrice l'assimilation de l'école maternelle «à une garderie»). Dans la mesure où les enfants ne peuvent venir seuls à l'école, cette deuxième catégorie de "présence » est toujours un discours sur ce qui est perçu de l'investissement parental dans l'école. Enfin, la présence physique des parents eux-mêmes joue comme outil de classement et d'évaluation sociale.

Dire d'un parent d'élève qu'il est « très présent » revient à célébrer à la fois son investissement dans l'éducation de son enfant et sa relation à l'école et de ce fait à l'identifier à la fois comme bon parent et comme bon parent d'élève. L'observation de ce type de jugements fait apparaître leur inscription sociale : ce sont les mères de classes moyennes qui sont jugées " très présentes ", par opposition à la fois aux mères d'origine populaire et aux mères de classes supérieures, parfois perçues comme trop « distantes ». Inversement, les parents « qu'elle ne voit jamais », qui ne sont " jamais là »", c'est-à-dire en fait qui ne lui parlent pas, appartiennent aux classes populaires. L'absence d'aisance sociale face à l'institution scolaire et à sa représentante est alors retraduite dans un espace normatif à la fois scolaire et familial (être un mauvais parent d'élève revient à être un mauvais parent). Ces présences ou absences parentales ne sont jamais plus évidentes que dans leur manifestation spatiale : les parents d'origine populaire entrent rarement dans la classe lorsque l'institutrice s'y tient, et attendent le plus souvent les enfants dans le couloir, alors que les parents de classes moyennes ou supérieures occupent bien plus fréquemment le «foyer » social que constitue la salle de classe. Ils deviennent alors de fait les destinataires privilégiés de la mise en scène de l'excellence pédagogique de l'enseignante sur les murs de la classe (panneaux servant à expliciter et exposer le travail réalisé lors du regroupement, exposition des travaux d'enfants, etc...), et des interlocuteurs fréquents et informés.

11. Il s’agit moins ici d’un jugement « de genre » (qui tendrait à reprocher à une mauvaise mère de préférer son travail à son enfant) qu'un jugement de classe : une mère de classe moyenne qui travaille, mais trouve le moyen de se libérer pour la soirée « portes ouvertes », et à cette occasion parle longuement avec l'institutrice, peut tout à fait ne jamais venir chercher son enfant, elle ne sera pas décrite comme "absente ». C'est bien une aisance vis-à-vis de la chose scolaire qui est codée dans cette catégorie sociale de la " présence ». En même temps, cette norme de la bonne présence est dirigée quasi-exclusivement sur les mères, qui de fait constituent la quasi-totalité des parents d'élèves que l'institutrice rencontre. 
Ainsi, comme le remarquait une institutrice de l'école à propos des enfants d'origine étrangère, observation que l'on peut élargir aux enfants d'origine populaire : "Ces enfants-là, il ne faut pas les scolariser comme des enfants scolaires ». Comment interpréter cette phrase sans y voir l'imbrication extrême des socialisations familiales et scolaires? Une certaine forme de socialisation familiale (la "bonne forme ", pour l'enseignante) scolarise déjà l'enfant. L'enfant " bien " socialisé est prédestiné au scolaire, déjà scolaire, déjà scolarisé. Ce type de discours est donc un discours d'impuissance scolaire : loin de l'idéologie méritocratique qui affirmerait la puissance de l'école dans sa capacité à scolariser des « enfants », indépendamment de leur origine sociale, il semble reconnaître que l'école ne scolarise que les enfants « déjà » scolarisés, les familialement scolarisés.

\section{LA PSYCHOLOGISATION DES DIFFERENCES SOCIALES}

C'est à travers un lexique psychologique que ces différences et ces identités sociales sont exprimées, évaluées, et naturalisées. On peut le mettre en lumière en considérant les deux extrêmes de l'échelle des évaluations indissociablement sociales et scolaires : le " club des chipies ", du côté de l'excellence, et le " cas » Grégory, de celui de la relégation.

\section{EXCELLENCE SCOLAIRE, EXCELLENCE SOCIALE}

"Le club des chipies » : c'est ainsi que l'institutrice désigne le groupe formé par Juliette (père ingénieur, mère secrétaire de rédaction), Honorine (père ?, mère secrétaire au quai d'Orsay) et Lara (père responsable informatique, mère sans profession $)^{12}$.

Tous les critères font système dans la définition de ce groupe comme celui des meilleures élèves ${ }^{13}:$ l'institutrice les cite lorsqu'elle évoque la " participation au regroupement et à la gym », la maturité, les compétences scolaires, mais aussi la tenue de soi et les façons de manger à la cantine par exemple ("Ça se

12. Les origines sociales des enfants ont été établies à partir des fiches remplies par les parents en début d'année. Elles sont, toutes classes confondues, réunies dans un classeur en salle des maîtres. On utilisera le point d'interrogation pour indiquer que la fiche ou qu'un renseignement manquait (ces absences de fiches ou d'indications ne sont pas distribuées au hasard, ils correspondent pour la classe à des parents que l'institutrice décrit par ailleurs - sans recours à la fiche donc - comme "défavorisés socialement »). Une exception cependant : les parents d'Honorine sont divorcés, c'est la mère d'Honorine qui a rempli la fiche où ne figurent ni le nom ni la pro fession du père.

13. « Meilleures élèves » des filles, mais également « meilleurs élèves » de la classe. Sur la question des performances scolaires des filles dans les petites classes, cf. Régine Sirota, $L$ École primaire au quotidien, op. cit., et, plus généralement, Christian Baudelot, Roger Establet, Allez les filles!, Paris, Seuil, 1992. 
recoupe ! ", dit-elle elle-même). Mais la définition et les manifestations de cette excellence se situent moins du côté du respect de la règle du jeu que de celui du « jeu avec la règle du jeu $»^{14}$. Malgré tous les appels de l'institutrice à l'obéissance et au calme des enfants, conditions de possibilité premières du travail pédagogique (il faut « tenir sa classe » si l'on veut pouvoir « travailler »), la désobéissance peut aussi, en effet, être positivement interprétée comme une marque de vivacité ou le signe de la participation scolaire. Dans ce contexte, elle peut être acceptée, voire encouragée, comme on le voit dans la scène suivante. En décembre, Honorine est malade, elle n'est venue en classe que le dernier jour, pour la fête du Père Noël. Son " calme » a été tout de suite relevé par Corinne, «l'ASEM » (Agent Spécialisé d’École Maternelle) :

_ Aujourd'hui, Honorine, on voit qu'elle est malade, elle est toute calme la pauvre.

- (moi) Pourquoi, elle est pas comme ça d'habitude?

- Oh non!

- (moi, en riant) C'est une peste ?

- Non, non, pas du tout, mais elle est un peu comme Juliette, quoi, c'est le même genre (nous soulignons).

Honorine est donc « du même genre » que Juliette. On peut faire l'hypothèse que sexe et origine sociale élevée se conjuguent dans l'appréciation de ce "genre » qui permet alors à Honorine de n'être pas « une peste » malgré sa désobéissance ${ }^{15}$. Le fait qu'elle soit « calme », loin d'être valorisé, est une source de regret, alors que l'injonction au calme (pour limiter «l'énervement » des enfants... et la fatigue de la maitresse) est une constante dans la classe. Ces manquements au calme et à l'obéissance ne peuvent être perçus, par l'institutrice ou l'ASEM mais aussi par les enfants, que sur le mode de l'exception. Exception dont bénéficient alors Juliette, Honorine, et Lara. Les termes employés pour désigner certains des enfants montrent bien comment s'organise l'acceptation de leur désobéissance. Le terme de "peste », jugé trop négatif, a été refusé par Corinne, et Catherine, l’institutrice,

14. «Il n'est pas de société qui ne propose, outre des modèles de conduite codifiés ou non, des modèles de modalité de la conduite accomplie et exemplaire, modèles régissant la manière d'exécuter les modèles, règles régissant la manière d'obéir aux règles, ou de leur désobéir : au jeu de l'excellence. le jeu avec la règle fait toujours partie de la règle du jeu " (souligné par nous), Pierre Bourdicu, Monique de Saint-Martin, L'Excellence scolaire et les valeurs du système d'enseignement français, texte ronéoté, CSE, 1969, cité in Régine Sirota, L'École primaire au quotidien, op. cit., p. 66.

15. Il est possible que cette désobéissance ne soit alors pas condamnée parce que perçue comme un signe paradoxal d'intégration au scolaire : « Ainsi quand les enfants de cadres supérieurs/professions libérales bavardent aussi facilement, ils manifestent en même temps leur intégration scolaire et la distance avec laquelle ils la vivent ", Régine Sirota, L'École primaire au quotidien. op. cit., p. 163. 
emploie fréquemment l'expression bien plus indulgente "Honorine la coquine » pour sanctionner (tout en approuvant implicitement) ses " bêtises " ou désobéissances : "Tu es bien Honorine la coquine, toi ».

Pour les enfants qui en bénéficient, la perception de l'indulgence professorale peut donner lieu à la revendication d'un véritable droit à la désobéissance. Ainsi, Juliette ou Honorine se permettent nombre de manquements à la règle édictée, souvent accompagnés d'une sorte de prise de distance par rapport à ces derniers, qui témoigne à la fois d'une connaissance de la norme et d'une volonté de s'en distinguer. Lors d'un exercice de vélo, par exemple, Honorine fait mine de sortir de l'itinéraire tracé en contournant les plots, puis finalement suit la consigne ; Juliette s'assoit sur la chaise de Catherine, l'institutrice, lors du regroupement, avec un sournre, et attend les remontrances.

Quant à Lara, bien que plus jeune, elle fait très fréquemment usage de ce droit à la désobéissance. À deux ans et demi, elle paraît parfaitement capable de sentir le privilège que représente le capital social de sa mère (qui est une « amie » de la directrice), et qui se manifeste par la présence légitime de cette dernière dans la classe et ses discussions avec l'institutrice. Elle utilise ce privilège comme un recours possible contre l'autorité pédagogique, ainsi qu'en témoignent les exemples suivants. Dès la première semaine de terrain, Corinne l'ASEM m'avait prévenue : « Elle en fait un peu à sa tête, il faut dire que ses parents sont des amis de la directrice. Elle est mignonne et en plus elle est pas bête, elle a bien compris le truc : quand on l'embête elle dit "je veux voir Mme B* (la directrice) ". Ainsi, en décembre, Lara dérange la cuisine, volontairement. Comme Catherine la reprend, elle lui répond « Je le dirai à maman que tu m'embêtes ». Catherine réagit fortement, en lui faisant les gros yeux tout en annonçant : " Non, c'est moi qui le dirai à ta maman ». En mars, la mère de Lara, qui est venue l'accompagner, discute longuement avec Catherine dans la classe. Lors de la matinée qui suit, Lara désobéit fréquemment aux injonctions de Catherine, qui la prend à part et lui dit d'un ton très sérieux : « Lara, je sais ce qui se passe dans ta tête. Ce n'est pas parce que je parle avec ta maman que tu peux faire tout ce que tu veux ensuite dans la classe ». La violence de cette réaction, à l'inverse de l'indulgence avec laquelle sont acceptées les « coquineries » d'Honorine ou de Juliette évoquées plus haut, peut sans doute s'expliquer par les différences de nature de ces déviances supérieures. La conduite de Lara rend l'avantage social visible en tant que tel : il met de ce fait davantage en cause l'autorité pédagogique de l'institutrice que les attitudes plus subtiles (peut-être plus « mûres ", Lara étant la plus jeune des trois) de Juliette et Honorine. En effet, on a affaire dans le cas de Lara à l'utilisation explicite d'un capital social, et non à la manifestation d'un capital culturel.

" Coquines », « chipies », des petites filles « qui n'en font qu'à leur tête » mais qui sont « mignonnes » et « pas bêtes », ces catégories rendent compte d'un rapport 
spécifique à l'école, fait d'intégration et de distance par rapport à la règle du jeu scolaire. À travers ces désignations individuelles et psychologiques, c'est la prise en compte et la valorisation d'une aisance vis-à-vis de la chose scolaire qui transparaît.

\section{CODAGE COLLECTIF ET HOMOGAMIE ENFANTINE}

Le codage par groupe d'enfants (l'image du " club») rend bien compte du fait que cette perception se fait moins à partir d'attributs individuels que par identification de compétences sociales partagées. Ainsi, ceux qui sont toujours ensemble peuvent être considérés et désignés de façon collective, et l'homogamie sociale précoce que l'on peut observer à l'école maternelle amplifie les relations entre origine sociale et codage scolaire. De plus, il n'est pas impossible que les enfants perçoivent certains de ces processus, comme on peut le supposer au vu de la scène suivante : Honorine et Juliette sont côte-à-côte en train de dessiner, Zina (père salarié dans un hôtel, mère assistante maternelle) est en face d'elles et dessine également. Zina est très active au regroupement, elle "participe " très bien selon l'institutrice. L'institutrice la complimente souvent, mais ne la cite pas au même titre que Juliette ou Lara quand elle évoque ceux qui participent le mieux. Zina montre son dessin à Juliette en lui demandant : "C'est beau, dis, Juliette ? ". Juliette lui répond : «Oui, c'est beau ». Ce type de demande (celle du jugement esthétique d'un autre enfant) n'est pas très fréquent en classe. Il est de ce fait significatif qu'elle se produise ici, et dans ce sens-là, de Zina à Juliette, élève modèle donnée en exemple aux autres. Zina ajoute ensuite : "C'est beau, alors t'es ma copine? ». Or, ce que l'on peut décrire comme une tentative de reconversion d'un jugement d'affinité culturelle en capital social échoue, Juliette répond « Non » et Honorine ajoute « C'est moi sa copine ».

J'ai tenu un relevé assez strict des fréquentes déclarations d'amitié ( C'est ma copine », «c'est mon copain », « t'es ma copine... ») et des relations entre enfants (qui étaient toujours ensemble, qui se voyaient hors de l'école, qui dansaient ensemble lors des fêtes ou de la gym). L'homogamie sociale est d'autant plus forte qu'elle est relayée par de nombreux mécanismes, aussi bien internes à la classe (l'institutrice peut apparier des enfants qui lui paraissent aller ensemble, le «club des chipies » se rassemble de lui-même autour des livres d'images...) qu'externes (la sociabilité enfantine étant organisée par la sociabilité parentale, les mères qui se connaissent ou se voient en dehors de l'école pouvant par exemple venir chercher alternativement les enfants ou les garder le mercredi).

Cette assignation d'identité par le groupe se fait autant en direction des «bons " (le « club des chipies ») que des " mauvais » élèves : « on a récupéré le bon lot aujourd hui » (nous soulignons) annonce par antiphrase Corinne, l'ASEM, 
devant Rodcharmie (père maçon, mère agent de propreté), Kevin (père commercial, mère au chômage, auparavant secrétaire), Bryand (?), Grace (?) et Élodie (?).

Ainsi, la personnalisation du rapport de l'institutrice aux enfants dont témoigne l'usage d'un vocabulaire psychologique visant à apprécier des caractéristiques personnelles ( « coquine » par exemple) s'inscrit paradoxalement dans une entreprise de codage collectif, qui à la fois manifeste et contribue à construire une homogamie sociale précoce.

\section{LE CAS GRÉGORY : DE LA * NON-PARTICIPATION 》 AU * RETARD MENTAL *}

Si l'on a choisi de présenter dans ce qui suit non pas un groupe de "mauvais élèves », mais bien un enfant, c'est parce que l'étude de ce cas manifeste également l'effet structurant (et non pas seulement structuré) des catégorisations indigènes et leur caractère dynamique, ainsi qu'un usage de la psychologisation des caractéristiques sociales.

Grégory (père transporteur routier, mère sans profession) fait partie de " ceux qui ne participent pas ». Cette catégorie, bien plus euphémisée que la catégorie de « ceux qui participent bien », ne s'observe pas d'emblée. L'absence de ces enfants dans les interactions avec l'institutrice se manifeste par une double négation : ils ne sont ni félicités, ni grondés. Au cours de l'enquête, ma prise en compte de ces « non-personnes » (au sens goffmanien du terme) n'a été effectuée qu'à partir d'une confrontation entre les noms qui apparaissaient dans le journal de terrain (cités par l'institutrice ou évoqués par moi) et la liste de la classe. Ce n'est que dans un deuxième temps que j'ai appris à reconnaître et plus généralement à observer ces enfants, et que j'ai pu solliciter le jugement de l'institutrice sur eux. Cette démarche permet de proposer une analyse des processus sociaux de construction d'un type particulier de «mauvais » élève. Le terme de " construction » s'impose ici, ne serait-ce que parce que la progression de cette construction a pu être perçue comme telle entre décembre et mars, les deux périodes d'observation.

En décembre, Grégory est décrit comme « ne participant pas ", « ni au regroupement, ni à la gym » : «il faut toujours venir le chercher ». C'est donc la nonparticipation qui définit le " mauvais » élève dans ce contexte - et non la désobéissance qui, on l'a vu, peut, dans certains cas, être valorisée. Une conduite non-participative n'est pas une conduite " sage » mais une conduite apathique : Grégory est souvent le premier à obéir quand l'institutrice demande d'aller s'asseoir pour le regroupement ou de se mettre en rang pour aller aux toilettes. Mais loin d'être vue comme " obéissante ", sa conduite, qui est associée à un retrait de participation constant (il ne participe pas aux discussions pendant le regroupement, se tient souvent à l'écart des autres dans la classe, tout en étant attentif à ce qui s'y passe), est traduite par ce qui apparaît comme le jugement négatif par excellence : 
«Lui, il ne fait rien », « il n’y a rien à en tirer ».

En mars, l'institutrice fait l'hypothèse qu'il s'agit de quelque chose comme un « retard mental ", lié aux relations entre Grégory et sa mère. Ainsi m'expliquet-elle, pour justifier le fait qu'elle a donné un instrument de musique à tous les enfants, mais pas à Grégory, lors du regroupement du matin :

C'est parce qu'il a encore fait pipi dans sa culotte. Tu comprends, j'en ai assez, c'est systématique maintenant à onze heure et demie. Alors moi je ne m'en occupe plus, parce que c'est juste avant l'heure des mamans, donc c'est clairement un problème avec sa mère, pas avec l'école. C'est fou parce que sa mère me dit qu'il est propre depuis qu'il a deux ans, et elle dit qu'à la maison il dessine, il chante des chansons... Alors je sais pas si c'est un problème avec moi, si je le bloque ou pas... ou bien plutôt si c'est parce qu'elle ne veut pas me dire qu'il est un peu retardé (nous soulignons).

L'institutrice fait ainsi jouer la réalité de l'expérience scolaire contre le discours familial : c'est elle qui sait s'il est pensable que, à la maison, il soit propre, qu'il « dessine ", qu'il « chante des chansons ». De plus, l'utilisation des catégories et des explications psychologiques (le problème avec la mère expliquant l'incontinence de fin de matinée, et l'hypothèse du retard mental) permet de réinscrire le comportement de Grégory dans la sphère familiale. Sphère familiale que l'institutrice décrit par ailleurs comme très démunie financièrement puisque, demandant aux mères d'élèves d'apporter des gâteaux pour la fête de Noël, elle en a dispensé la mère de Grégory : "Je crois qu'ils n'ont pas beaucoup d'argent, donc je ne lui ai pas demandé ". Les fonctions de cette réinscription du comportement de Grégory dans la sphère familiale sont de deux ordres : tout en permettant à l'institutrice de réfuter l'hypothèse d'une responsabilité personnelle et professionnelle face à ce " cas à problème » («je sais pas si c'est un problème avec moi, si je le bloque ou pas... ou bien plutôt si c'est parce qu'elle ne veut pas me dire qu'il est un peu retardé »), ce discours concourt également à maintenir la possibilité d'un discours « méritocratique » sur l'école maternelle ( C'est un problème avec sa mère, pas avec l'école »)".

Qui plus est, l'incontinence enfantine donne rarement lieu à une punition, les enfants pouvant être grondés, mais non punis. Dans le cas de Grégory, la privation d'instrument de musique n'est pas un événement unique :

Je ne sais pas ce qui se passe, avec cette histoire de pipi dans la culotte. Au début de l'année, je ne l'ai pas puni, parce que je ne punis pas vraiment, tu as vu, mais

16. Dans le cas de Grégory comme dans l'expression évoquée plus haut sur les « enfants non scolaires ", on note un même type d'extériorisation qu'on peut interpréter comme la gestion d'un échec qui serait tout autant celui de l'institutrice (en tant que personne et en tant que représentante de l'institution scolaire) que celui de l'élève. 
bon, je le mettais à l'écart quoi, parce qu'à l'école il faut des règles, il faut qu'il comprenne qu'il y a des règles (nous soulignons).

Il est tout à fait possible que Grégory ait suffisamment « bien » intériorisé la légitimité de cette " mise à l'écart " répétée pour la reproduire de lui-même, renforçant ainsi sa non-participation.

Entre les deux séquences d'observation, l'institutrice est donc passée du reproche de non-participation à la découverte d'un retard. Or, on peut voir dans cette dynamique de la catégorisation l'effet de l'étiquette originaire attribuée à Grégory. En effet, une pratique identique (l'incontinence enfantine, par exemple) n'est pas envisagée de la même façon selon les enfants. Pour les enfants « actifs ", " ceux qui participent », elle est bien souvent perçue comme le pendant de leur agitation. En revanche, elle devient plus grave pour Grégory du fait que, par ailleurs il ne " participe pas ». Si on prend en compte la façon dont il a été puni, on peut risquer l'hypothèse du caractère structurant des catégorisations et de la construction d'un retard mental. De plus, la solution préconisée par l'institutrice (« la mère devrait l'emmener chez le psychologue ») pourrait constituer un marquage supplémentaire dans sa scolarité ultérieure.

\section{LES USAGES DE LA PSYCHOLOGISATION DES DIFFÉRENCES SOCIALES}

Les processus sociaux de construction tant du « bon » que du « mauvais » élève de maternelle font donc intervenir des catégories et des explications psychologiques. Cette psychologisation du jugement et de l'évaluation constitue un levier historique de la professionnalisation (et un élément de la compétence professionnelle) des enseignants d'école maternelle, et il faut prendre ce fait en compte si on ne veut pas passer à côté de l'enjeu que l'usage de ce registre psychologique représente pour l'enseignante : l'histoire de l'école maternelle montre, en effet, à quel point sont liées l'élaboration d'un savoir sur la petite enfance et la professionnalisation des enseignants ${ }^{17}$.

17. * Les ambitions scolaires de la première éducation publique et l'interprétation savante de l'hygiène et de la pédagogie ont transformé les maîtresses des plus petuts en véritables institutrices (...) La découverte scientifique de la fécondité de la "petite enfance", selon l'expression de Jean Piaget, renforce la légitimité et l'audience de l'enseignement qui lui est destiné », Jean-Noël Luc, * De la gardienne à l'institutrice d'école matemelle. La professionnalisation de l'éducation publique des jeunes enfants au XIX" siècle ", in Pierre Guillaume (dir.), La Professionnalisation des classes moyennes, MSHA, 1996, (pp. 181-193), pp. 189-191. Dans « Le métier d'enfant... ", loc. cit., Jean-Claude Chamboredon et Jean Prévot notent le même phénomène et analysent la « transformation du métier d' "institutrice" en métier d" "éducatrice", réinvention ou anoblissement ", à travers la production « d'agents disposés à l'introduction de disciplines nouvelles (psychologie, psycho-pédagogie, mathématiques modernes) et d'une définition nouvelle de l'enfance, agents intéressés à la fois par et à cette transformation dans la mesure même où ces changements de contenu sont liés à une modification du sens de la profession qu'ils exercent » (pp. 317-318). 
Par ailleurs, c'est bien la naturalisation et la personnalisation de dispositions sociales qui se jouent dans l'usage de ce lexique psychologique et dans le regard que l'institutrice porte alors à la fois sur parents et enfants's . Ce qu'on peut ajouter ici, c'est que cette psychologisation des jugements sociaux est susceptible d'usages multiples selon le public concerné par les catégorisations. Dans le cas de Grégory, l'invocation du lexique psychologique permet la stigmatisation de la socialisation familiale à travers le diagnostic d'une pathologie. La psychologisation a également pour effet de décharger l'école de la responsabilité de ce "cas à problème ». Mais elle peut avoir d'autres fonctions, ou effets, lorsqu'elle s'applique aux enfants d'origine moyenne ou supérieure. En affinité avec leurs socialisations familiales, elle peut être un instrument de valorisation de celles-ci : à travers par exemple les normes partagées sur l'appréciation des travaux et surtout des dessins d'enfant ${ }^{19}$ et les pédagogies inductives inspirées de la psychologie de la petite enfance où s'illustrent particulièrement les enfants d'origine moyenne ou supérieure. Mais elle peut également servir à excuser leurs défaillances : par exemple dans le cas d'Honorine, membre du " club des chipies ", qui n'arrive pas à lire son prénom, l'institutrice précise que " les psychanalystes disent que les enfants passent par des stades de régression du savoir ", qui ne gênent en rien, au contraire, leur développement intellectuel futur. Cet usage dédramatisant de la psychanalyse n'a été observé que dans ce cas. On peut d'ailleurs noter la différence entre le lexique employé pour parler de Grégory, plutôt du côté d'une vulgate psychologique assez répandue (il est « retardé », l'incontinence est « un problème avec sa mère »), et cette utilisation d'un lexique de forme plus technique, défini comme "psychanalytique " ("stades de régression du savoir »). La différence de contenu entre les deux discours est donc redoublée par la légitimité différente de leur référence (psychologie pour Grégory, psychanalyse pour Honorine). La psychologisation est donc susceptible d'usages multiples, tant dans les choix de lexiques que dans l'utilisation de ces choix.

18. Pour une analyse analogue sur le terrain de la Protection Maternelle et Infantile, $c f$. Delphine Serre, "Le "bébé superbe". La construction de la déviance corporelle par les professionnel(le)s de la petite enfance ", Sociétés Contemporaines, ${ }^{\circ} 1$, juill. 1998, pp. 107-127: « quand il s'agit des classes populaires, les professionnelles ne livrent jamais directement l'arrière-plan social des figures déviantes. Elles ont plutôt tendance à interpréter cet écart en termes psychologiques, ce qui a pour effet de personnaliser les causes des déviances ", p. 120.

19. Comme le notent Jean-Claude Chamboredon et Jean Prévot : «En somme, les conditions de compréhension des exercices de la maternelle découlent des conditions d'invention de ces exercices : la competence objectivement requise des parents n'est-elle pas, jusqu'à un certain degré au moins, la connaissance des théories, des méthodes, des mouvements intellectuels et artistiques qui ont rendu possible cette invention ? (...) Finalement, c'est le rapport tout entier des enfants aux apprentissages qui est, on peut le supposer, transformé par la bonne compréhension, par les parents, des apprentissages scolaires ", "Le métier d'enfant... », loc. cit., pp. 327-328. 


\section{LA SOCIALISATION COMME ENJEU : LA DISTINCTION DES AGENTS DE LA SOCIALISATION}

Parler de socialisation " par l'école ", c'est peut-être artificiellement réifier l'école comme instance de socialisation, là où l'analyse empirique fait apparaître une diversité d'agents et de processus. En première année d'école maternelle, il y a au moins deux agents potentiels de socialisation : l'institutrice, agent évident, et l'ASEM, « agent spécialisé d'école maternelle ", ancienne « femme de service ", statutairement chargée d'aider l'institutrice dans les aspects les moins pédagogiques de la vie quotidienne à l'école maternelle ${ }^{20}$. Cette pluralité des agents de la socialisation constitue un fait accessible à la seule observation : là où dans les textes officiels, à la rubrique « enseignement-éducation », il n'est fait référence qu'à l'institutrice, à l'heure des ateliers dans une classe de première année de maternelle, il y a en fait deux personnes qui s'occupent des enfants.

\section{LA LEGITIMITÉ À SOCIALISER}

Une sociologie de l'école maternelle réduite à une sociologie des instituteurs est ainsi insuffisante à rendre compte du processus de socialisation qui s'y déroule. En termes de temps passé avec les enfants, de présence symbolique ou effective, de rôle dans le processus éducatif scolaire, l'ASEM doit être étudiée au même titre que l'ınstitutrice. L'observation conduit à une rupture avec le point de vue institutionnel : en effet, les textes officiels qui décrivent la présence de l'ASEM sont sans ambiguité. On a d'une part une description du travail noble et légitime - le travail " pédagogique » et socialisateur de l'institutrice -, et d'autre part celle d'un contact utilitaire, subordonné, corporel, un " sale boulot » au sens d'Everett Hughes ${ }^{21}$. Cette différence apparaît dès la porte de la salle de classe, côté couloir, où sont affichés d'une part un carton blanc où est calligraphié " Classe de Mme X, Catherine » et d'autre part une feuille ayant servi aux enfants à faire des dessins et retournée, « Corinne, notre ASEM, chargée de l'hygiène et de la propreté des enfants et de la classe. "

Les débuts de l'école maternelle avaient déjà été placés sous le signe de l'alternative entre « assistance » ou garde et «éducation $»^{22}$. Cette alternative historique se retrouve aujourd'hui de manière transversale dans les statuts et les rôles assignés respectivement à l'ASEM et l'institutrice. Tout se passe donc, dans les textes officiels,

20. Sur le statut institutionnel des ASEM, cf. Bernard Lahire, La Raison des plus faibles : rapport au travail, écritures domestiques et lectures en milieux populaires, Lille, PUL, 1993, chap. 2.

21. Cf. Everett Hughes, "Social role and the division of labor ", Midwest Sociologist, n 17 (1), 1956, pp. 3-7 (pour une trad. en français, cf. Everett Hughes, Le Regard sociologique. Essais choisis. Textes rassemblés et présentés par Jean-Michel Chapoulie, Paris, Presses de l'EHESS, 1996, pp. 61-68).

22. Jean-Noël Luc, L'Invention du jeune enfant au XX siècle. De la salle d'asile à l'école maternelle, Paris, Belin, 1997. 
comme si la présence constante de l'ASEM dans la classe ne pouvait avoir aucun effet " pédagogique ", et comme si la distinction entre " accueil » et " pédagogie » était un fait acquis en première année de Maternelle et non le résultat d'un travail constant de négociation. À rebours, on peut choisir de prendre en compte la présence des ASEM et d'en rechercher les effets socialisateurs. La façon dont l'institutrice et l'ASEM se comportent face aux enfants, les modèles éducatifs qu'elles leur proposent implicitement ou explicitement s'inscrivent en fait dans leur propre système de représentations et de pratiques, lié à leur position dans l'espace social.

Corinne, l'ASEM, a vingt cinq ans, elle est d'origine populaire, et sans qualification professionnelle. L'année de l'enquête, elle prépare le CAP « Petite enfance », ce qui provoque un double discours de la part de l'institutrice et de la directrice : tout en l'encourageant à obtenir le diplôme, voire en l'aidant concrètement, institutrice et directrice n'en insistent pas moins souvent sur le fait que ce diplôme n'aura aucun effet sur le monopole du travail pédagogique détenu par les institutrices :

Avec ça, elles [les ASEM] croient, enfin, je les accuse pas, on leur fait croire, qu'elles pourront devenir quelque chose comme des adjointes d'institutrices. Alors c'est sûr, ça leur monte la tête. Avec ça, faudrait savoir, si elles deviennent des institutrices, nous on va faire quoi? Laver par terre? Elles ne vont plus rester à leur place.

Catherine, l'institutrice, vient d'une famille de classe moyenne ; elle est mariée à un cadre de l'aéronautique. Ces différences d'origine sociale et de positions professionnelles entre institutrice et ASEM se traduisent dans des modèles éducatifs différents, dont on peut citer quelques exemples. Tout d'abord, ce qu'on pourrait nommer les « pratiques d'obéissance » (comment on demande et/ou obtient l'obéissance). Là où l'ASEM utilise le corps (elle va chercher les enfants, les prend par le tee-shirt pour les rasseoir, ou leur prend l'objet des mains), l'institutrice a plus souvent recours à la parole (elle les interpelle et leur demande d'arrêter d'embêter leurs camarades, de lâcher tel ou tel objet, de venir...). L'ASEM emploie plus fréquemment la punition en cas de crise (le « coin »), là où l'institutrice va mettre en scène le fait qu'elle raisonne (par la parole, donc) les enfants. On retrouve ici les traits des " pédagogies différentielles » des classes populaires et moyennes-supérieures : l'autorité stricte d'un côté, la « pédagogie anti-autoritaire » inspirée des « nouvelles normes psycholo-

23. Sur les différences dans les pratiques éducatives, voir par exemple François de Singly, « Les ruses totalitaires de la pédagogie anti-autoritaire ", Revue de l'Institut de Sociologie, n 1-2, 1988. pp. 115-126 et Jean-Claude Passeron, François de Singly, "Différences dans la différence : socialisation de classe et socialisation sexuelle ", Revue Française de Sciences Politiques, ${ }^{\circ} 1$, 1984, pp. 48-78. Pour une analyse convergente des différences, à l'école maternelle, entre institutrice et " femme de service " sur cette question de l'autorité, of. Geneviève Dannepond, "Pratiques pédagogiques et classes sociales... », loc. cit., p. 45. 
giques » sur le développement de l'enfant, de l'autre"'. Mais une telle différence témoigne également de la division institutionnelle (intériorisée ou mise en pratique) entre " garde » et " éducation ".

De plus, il y a chez l'ASEM une affirmation pratique plus forte des différences de " genre » entre les enfants. On peut prendre ici l'exemple des jouets comme l'un des marqueurs forts de l'identité sexuée, et d'une scène très classique en maternelle : deux enfants se disputent un jouet. Lorsqu'ils sont de sexe différent, Corinne, l'ASEM, a tendance à trancher alors la propriété temporaire par le genre. S'il y a conflit entre un garçon et une fille au sujet d'une casserole, elle est donnée à la fille. Si c'est au sujet d'une petite voiture, elle est donnée au garçon. L'institutrice, en revanche, est apparue comme plus neutre par rapport à ces catégories. Cependant, cette différence entre institutrice et ASEM peut aussi se comprendre comme un artefact d'observation. La présence de l'enquêtrice signifie ici beaucoup pour l'institutrice, instruite par son cursus d'étude des discours sociologiques ou psychosociologiques sur l'importance de la prime éducation dans la construction des différences de genre et le caractère souhaitable d'une égalisation des pratiques éducatives. Elle peut donc avoir veillé à ne pas enfreindre cette norme en ma présence, là où pour l'ASEM la présence d'une sociologue ne représente pas forcément de norme en la matière ${ }^{24}$.

Enfin, une différence particulièrement notable existe dans la division du travail de relation aux parents. Les enquêtes sur l'école primaire ont fait apparaitre que les instituteurs reprochaient souvent aux parents d'origine populaire leur absence de la classe, mais que ce qu'ils traduisent comme un désintérêt de la part de ces parents pour la scolarité de leurs enfants peut provenir du rapport tendu que ces derniers entretiennent à l'égard de l'institution scolaire et de ses représentants ${ }^{25}$. La caractéristique de l'école maternelle sur ce point pourrait tenir au fait que ces parents d'origine populaire trouvent en l'ASEM une interlocutrice plus proche d'eux socialement, et à qui ils parlent (ce qui invalide la thèse sociale du désintérêt pour la scolarité). L'institutrice a souvent évoqué devant moi tel ou tel parent qu'elle « ne voyait jamais », qui « n'entrait jamais dans la classe ». Or, j'ai retrouvé ces mêmes parents en train de discuter de la scolarité de leur enfant avec l'ASEM avant l'arrivée de l'institutrice le matin.

24. Sur l'inculcation des identités de genre et le contrôle des attitudes corporelles des garçons et des filles pour une même classe d'âge aux États-Unis, $c f$. l'enquête ethnographique de Karin A. Martin, « Becoming a gendered body : practices of preschools ", American Sociological Review, vol. 63, 1998, pp. 494-511.

25. Sur le " mythe de la démission parentale ", cf. par exemple Bernard Lahire, Tableaux de familles. Heurs et malheurs scolaires en milieux populaires, Paris, Seuil, 1995, pp. 270-273 ; et Régine Sirota, L'École primaire au quotidien, op. cit., chapitre VI. 


\section{LE TRAVAIL DE REIMPOSITION D'UNE DOMINATION}

Parler de socialisation scolaire sans préciser qui sont les agents de cette socialisation et les processus précis et divers qu'ils mettent en œuvre, c'est occulter la diversité des acteurs de la socialisation. Mais c'est aussi masquer le fait que cette diversité est conflictuelle, c'est faire l'impasse sur les luttes de classement et les conflits entre les différents agents " socialisateurs". Lors de l'enquête, un conflit violent, ayant pour enjeu le monopole du travail pédagogique détenu par les institutrices et le droit au contact " noble " avec enfants et parents, a opposé dans un premier temps Corinne et Catherine puis institutrices et ASEM à l'échelle de l'école. Les réunions houleuses (entre institutrices et directrice, ou institutrices, directrice et ASEM) qui ont eu lieu sur ce thème étaient particulièrement concentrées sur les catégories de "propre " et de "sale ", qui renvoient selon Mary Douglas à l'ordre et au désordre social ${ }^{\text {ha }}$. Dans le conflit entre institutrices et ASEM, il semble y avoir un usage réversible de l'accusation de saleté : les institutrices utilisent la catégorie (« cette école est sale») pour montrer que les ASEM ne font pas leur travail. En retour, les ASEM peuvent, en déclarant « vous nous avez laissé une classe très sale à nettoyer ", mettre en doute la capacité d'une institutrice à « tenir sa classe » (lors des ateliers de peinture par exemple) sans leur aide.

En rapprochant la violence de ce conflit et la menace que constitue l'élévation de la compétence professionnelle des ASEM (« elles ne vont plus rester à leur place »), on peut faire l'hypothèse que le travail de construction de la légitimité professionnelle des enseignantes se joue aussi dans les interactions quotidiennes avec les ASEM, et que de tels moments de réimposition d'une domination institutionnelle sont peut-être périodiquement nécessaires.

\section{TRAVALL D'EQUIPE ET DISTINCTION}

La violence symbolique et pratique de ce conflit vient également de la façon dont jugements et dépréciations professionnels et personnels sont constamment imbriqués ${ }^{27}$. Au premier plan du discours de Catherine sur Corinne apparaissent des accusations professionnelles et institutionnelles (Corinne « travaille mal » parce qu'elle veut faire " ce qu'elle n'a pas à faire », comme « parler aux parents », « leur donner les dessins des enfants », « préparer les découpages » et que du coup « la

26. Mary Douglas, De la souillure : études sur la notion de pollution et de tabou, Paris, La Découverte, 1992 (1967).

27. Ma position d'enquêtrice (j’étais plus perçue comme une « institutrice stagiaire » que comme sociologue) rend compte du fait que mes seules données sur ce conflit relèvent du point de vue de l'institutrice. En effet, au regard des tensions qui existaient, ma position a rendu difficile tout entretien avec Corinne, l'ASEM, sur ce thème. 
classe est dégueulasse "). Mais ce qui se joue aussi, et peut-être surtout, c'est une accusation sociale qui est à la fois une façon de prendre en compte l'action socialisatrice possible de l'ASEM et une volonté de se distinguer d'elle :

Surtout que tu sais, sa manière de parler, c'est toujours brusque, toujours agressif... elle a un ton un peu marchande de poissons, quoi. Et puis c'est vrai, avec les enfants aussi... Par exemple ils répètent tous un truc qu'elle dit quand il y en a un qui renverse le lait, «t'en fous partout », alors t'imagines... En plus c'est Honorine qui le répète, alors que chez elle ils sont super stricts sur le langage, la façon dont elle parle. Alors sa mère, ça va, je me fais pas de soucis elle sait que ça vient de Corinne si Honorine le dit chez elle, mais ses grands parents sont encore plus stricts que ses parents, alors si elle le dit chez eux... (nous soulignons).

On voit bien ici comment la volonté de se distinguer des comportements populaires à travers une mise en accusation est exacerbée par le fait que le travail de l'institutrice et de l'ASEM peut se donner à voir comme un travail d'équipe ou de couple, dont les résultats ne sont pas obligatoirement dissociables, par exemple pour la directrice, pour l'inspectrice ( "Si les tabliers sont sales et que l'inspectrice passe, c'est à moi qu'elle va le reprocher »), mais également pour les parents d'élèves. La violence du jugement social de Catherine sur Corinne s'inscrit donc également dans une nécessité de dissociation, de distinction par rapport à des regards extérieurs légitimes et dans la crainte d'un amalgame professionnel mais aussi « personnel », celui des « façons de parler » et des manières d'être.

La prise en compte des « invisibles sociaux " présente donc un avantage par rapport à la stricte assimilation de la socialisation scolaire à l'action éducative de la seule institutrice. Mais elle ne doit pas se faire en occultant les légitimités différentes des présences et des pratiques de socialisation des différents agents. Ce conflit sur le droit à socialiser, qui a d'abord concerné Catherine et Corinne puis s'est étendu à toute l'école, a finalement sanctionné « le monopole du travail pédagogique " détenu par les institutrices et s'est traduit par leur victoire et une réinstallation d'un partage des tâches fidèle à l'esprit des textes officiels. Institutrice et ASEM peuvent bien être toutes les deux agents de la socialisation, ce ne sont pas des agents « équivalents » socialement dans l'ordre de la légitimité à socialiser.

\section{LA SOCIALISATION COMME APPRENTISSAGE DES DISTINCTIONS : « ÉCOLE \# ET \& FAMILLE *}

À la variété et à la non-équivalence des socialisations familiales face à l'école maternelle s'ajoute donc la pluralité des agents de la socialisation scolaire. Mais la distinction même entre " école " et « famille " s'avère être le produit d'un apprentissage qui s'inscrit dans ces deux premiers systèmes de variation.

C'est à partir d'une recherche systématique des catégories indigènes de 
l'obéissance (réquisits explicites ou implicites, interdits, exhortations) que la question de l'apprentissage des distinctions entre « école » et « famille » a été travaillée. Le respect du « moment » est apparu comme l'une de ces catégories. Le terme de "moment » désigne un mixte de temps et de lieu, appuyé sur l'évocation d'une activité scolaire, que l'institutrice demande constamment aux enfants de respecter. "Qu'est-ce que le vendredi ? " demande t-elle aux enfants, qui lui répondent « le vendredi on va dans le dortoir et on regarde les diapositives ». Des expressions usuelles, comme "l'heure des mamans ", désignent à la fois une heure du jour ( $11 \mathrm{~h} 30$ ou 16h30), une activité (attendre l'arrivée des parents), et un lieu, le couloir. On est donc dans l'heure d'un lieu ou d'une activité, le terme de récréation étant beaucoup moins employé par les institutrices quand elles s'adressent aux enfants que l'expression «l'heure de la cour ». Le respect du moment, de la right thing in the right place at the right time, est un réquisit important de l'obéissance en maternelle. Alors que je suis dans la salle des maîtres, une enfant d'une autre classe vient dénoncer un camarade de classe à son institutrice : « Anthony il est dans l'heure des mamans et il met son manteau " (nous soulignons) - alors qu'il est seulement 10h30, «l'heure de la cour». Dans sa phrase, «l'heure des mamans » signifie « le couloir ». La désobéissance apparaît de ce fait comme le non-respect du moment, mixte de temps et de lieu.

Ce dernier réquisit de l'obéissance comprend alors une dimension très prescriptive quant à l'usage des objets. Un même objet ne doit pas être utilisé de la même façon selon son inscription temporelle et spatiale. Il en est ainsi des « bancs " en bois blanc présents dans toute l'école. Lorsqu'ils sont disposés en carré à l'intérieur de la classe pour le regroupement, ils s'appellent « les bancs » (Catherine aux enfants : "Quand on s'assoit sur les bancs, on n'a ni jouet ni doudou : on travaille ») : il faut s'y asseoir, et s'y tenir assis, et il est interdit de monter dessus. Lorsque ces mêmes bancs sont déplacés, pour servir aux ateliers par exemple, tout en restant dans la classe, on ne doit en principe pas monter dessus mais la règle est appliquée avec beaucoup plus d'indulgence. Sont permises en revanche toutes les utilisations ludiques des bancs, comme faire rouler des petites voitures dessus. Ils n'ont alors plus de nom («Tu descends de là ! »). Enfin, ces mêmes bancs dans le gymnase s'appellent « la maison » quand ils sont regroupés en carré (quand je dis aux enfants de monter sur « les bancs », ils me reprennent : « Non là c'est pas les bancs c'est la maison », et Catherine renchérit : "Les bancs c'est dans la classe, la maison c'est dans la salle de gym »), et il faut monter dessus (exercice d'équilibre). Il s'agit pourtant, bien sûr, des « mêmes » bancs à chaque fois. La socialisation apparaît bien ici comme un apprentissage, à la fois générique et spécifié, des " distinctions » et de la séparation symbolique entre ce qui est objectivement identique. Discours et pratiques d'obéissance ont donc également pour but l'intériori- 
sation de " catégories de l'entendement " à travers l'injonction au « respect du moment ". Apprendre à classer, à différencier, à distinguer - même l'objectivement identique - : ne peut-on faire l'hypothèse que se joue ici plus généralement l'apprentissage social d'un principe de vision et de division du monde ?

L'une des distinctions capitales dont l'apprentissage se joue ici est la distinction entre famille et école. Deux types d'indicateurs ont été utilisés pour l'observer. Tout d'abord, un relevé de toutes les injonctions de l'institutrice faisant intervenir une opposition entre le " chez toi " et "l'ici ». Ce type de phrase revient fréquemment lors des rappels à l'ordre des enfants ou de l'énonciation des règles en vigueur dans l'espace de la classe : "Chez toi " (tu peux faire ça) " mais pas ici » (nous soulignons). L'emploi de ce lexique de la rupture peut s'interpréter comme une façon d'affirmer, ou de réaffirmer, la distinction entre les espaces familial et scolaire. Par ce type d'injonctions, l'institutrice construit la légitimité d'un lieu où les pratiques familiales n'ont pas droit de cité absolu. En regard, il est intéressant de noter que l'ASEM, au contraire, utilise un lexique de la continuité : « Chez toi tu ne fais pas ça donc ici non plus » (nous soulignons). Il y a ainsi deux modèles différents des relations entre école et famille. Se joue ici bien évidemment la manifestation de l'intérêt professionnel plus grand de l'institutrice à affirmer une rupture entre les deux mondes et à construire, par là même, la spécificité et la légitimité de l'espace scolaire et du travail pédagogique. Mais on peut également faire l'hypothèse que Corinne, l'ASEM, est moins disposée que l'institutrice à disqualifier des socialisations familiales dont elle peut se sentir proche socialement, notamment lorsqu'il s'agit de reprendre des comportements qui mettent moins en jeu des questions d'enseignement que d'éducation ${ }^{28}$.

La distinction entre famille et école est donc explicitement affirmée et requise par l'institutrice. Mais elle s'incarne aussi, de façon plus subtile, dans l'organisation de l'espace même de la classe, ce qui constitue un deuxième type d'indicateur. La salle de classe observée se compose en effet de deux sous-ensembles distincts.

Un espace " école » tout d'abord, situé près de la porte, où l'on trouve le bureau de l'institutrice, ainsi que les tables pour les ateliers et les bancs du regroupement du matin. Un espace « famille » ensuite, au fond de la salle, où est reconstitué l'intérieur d'une maison, avec une chambre, un lit et des poupées, une cuisine et une table de cuisine, un salon et des livres. Sont donc matérialisées dans l'es-

28. Jean-Manuel de Queiroz décrit en effet ainsi les représentations populaires de l'école : « l'instruction constitue le seul domaine légitime des professionnels de l'enseignement et on [les familles populaires] en attend d'abord qu'il soit efficace, mais l'"éducation", la "bonne éducation", tout aussi importante, relève d'un domaine de compétence mixte, où les parents savent aussi bien et peut-être mieux que les enseignants ce qu'il convient de faire ". L'Ecole et ses sociologies, Paris, Nathan, 1995, p. 75. 
pace de la classe observée à la fois une réunion et une séparation de l'école et de la famille. Ces deux espaces sont différemment investis, pratiquement et symboliquement, par l'institutrice et l'ASEM. L'ASEM a beaucoup contribué à la mise en place de l'espace « maison », elle a cousu l'édredon du lit et les rideaux de la chambre. Elle y est très présente, beaucoup plus que l'institutrice ou que moimême, qui ai pris conscience de cette distinction en objectivant mes propres trajets à l'intérieur de la classe. Ces deux espaces correspondent d'ailleurs à deux types d'activités différentes : l'espace maison est un lieu non directement scolaire, un lieu de jeu libre et non dirigé, une sorte d'antichambre de la récréation. L'espace " école " est en regard un espace de travail. C'est l'espace des " ateliers ", où les règles de bonne tenue du corps par exemple sont fréquemment rappelées, où le temps est un temps « scolaire » très marqué, où l'on commence et finit les « travaux d'enfants ».

À partir de cette division spatiale et de ces différents usages de l'espace, on peut se demander dans quelle mesure la socialisation scolaire maternelle ne se construit pas en partie sur l'apprentissage de la rupture entre les mondes sociaux que sont la famille et l'école, et n'implique pas l'intériorisation de la légitimité scolaire. Cette organisation spatiale, à la fois structurée et structurante, et cette importance des murs et des meubles témoignent d'une organisation sociale et, en même temps, permettent son intériorisation. Dans la classe de maternelle comme dans la maison kabyle analysée par Pierre Bourdieu (tant dans ses oppositions internes que dans sa relation avec l'extérieur), l'important est que les deux sous-espaces « ne [soient] pas interchangeables mais hiérarchisés $»^{29}$.

Mais l'apprentissage de cette distinction et de cette hiérarchisation ne concerne peut-être pas tous les enfants au même titre. Le discours de l'institutrice sur la rupture entre les mondes sociaux que sont l'école et la famille semble bien plus insistant en direction des enfants d'origine populaire. Cette distinction serait donc plus fortement imposée aux enfants d'origine familiale « non scolaire », pour qui l'école serait construite comme un monde social radicalement " autre », alors que ce serait la continuité qui présiderait à l'établissement des relations entre école et famille pour les enfants d'origine sociale élevée. Bien qu'une telle idée n'ait qu'un statut d'hypothèse, elle suffit pourtant à ne pas faire conclure trop rapidement à un invariant (l'apprentissage d'une rupture entre école et famille) là où il peut y avoir des variations sociales.

Pour constituer la socialisation en objet sociologique, il faut donc tenir compte simultanément de trois variables : les destinataires de la socialisation, les agents socialisateurs, et les contenus de la socialisation. Pour les premiers, les enfants, la

29. Pierre Bourdieu, Le Sens pratique, Paris, Minuit, 1980, p. 460. 
socialisation varie en fonction des évaluations scolaires de leurs différentes socialisations familiales ${ }^{30}$. Pour les seconds, leur légitimité plus ou moins grande à socialiser, déterminée par l'institution scolaire, la division du travail quotidien de socialisation ainsi que les divers modèles de socialisation dont ils sont porteurs (selon leur propre socialisation professionnelle et familiale) déterminent leur rôle effectif dans la socialisation scolaire. Dès lors, les contenus de la socialisation, dernière variable, sont les produits des conflits autour de leur définition entre institutrice et ASEM et entre ces agents de la socialisation et les enfants. De plus, si ces contenus prennent essentiellement la forme de normes et de distinctions inculquées aux enfants, il est cependant très important de prendre en compte les sources et le langage normatifs à partir desquels celles-ci sont formulées. Le fait que la psychologie, par exemple, soit une référence privilégiée de la socialisation scolaire en maternelle a des effets très spécifiques : la psychologisation des différences sociales peut impliquer leur " pathologisation », leur naturalisation ou leur retraduction.

30. Il aurait fallu évoquer ici le fait que les parents sont aussi destinataires de la socialisation scolaire dans la mesure où s'effectue l'imposition d'un " métier de parent ". Elle se manifeste tout autant dans les interactions de face-à-face entre les parents et l'institutrice que dans le flux d'objets (dessins, objets fabriqués par les enfants) organisé par l'institutrice entre l'école et la maıson. L'étude de la socialisation en classe de maternelle ne se limite donc pas à celle d'une socialisation « primaire » qui ne concernerait que les enfants. 